"Field Trip Technique" the reader is told: "If transportation is to be provided ... every precaution must be taken to guarantee comfort and safety". "Leaders' Responsibility. It is helpful to plan how to keep attention centred on the main objective of the trip. . . . Leaders should speak distinctly enough for all to hear'". In describing a particular field excursion, negotiations for hiring the bus are given six lines; and three-quarters of a page is spent on reproducing, in entirety, the letter to parents asking approval for their children to go on the trip, and ensuring that they will be there to meet them on their return. In the same way, many of the photographs are no more than pleasant school groups taken in the field, telling the reader absolutely nothing.

Thus, while fully agreed that the teaching of conservation is a worthy cause, one finds it hard to recommend this book (with its inevitable high price) to teachers outside the United States.

MaUrice Ashby

\section{PROBABILITY AND STATISTICAL INFERENCE}

The Foundations of Statistics

By Prof. Leonard J. Savage. (Wiley Publications in Statistics.) Pp. xv + 294. (Now York: John Wiley and Sons, Ine.; London : Chapman and Hall, Ltd., 1954.) 48s. net.

M main objection to this book concerns its 1 title. The first half deals with theories of probability in their subjective and objective aspects ; the second half defines "statistics proper" as "the art of dealing with vagueness and with interpersonal difference in decision situations". Some of the words in this definition, perhaps, provide avenues of escape ; but as Prof. Savage goes straight into minimax theory, to which seven out of the closing ten chapters are devoted, it is fair to construe his attitude as one which takes statistics to be a theory of decision. This view is not shared by many British statisticians, who rightly insist, first, on the fact that statistical inference is only part of statistics and, second, on the fact that decisions are only part of statistical inference.

As a contributor to the foundations of the theory of decisions, Prof. Savage's book is, however, welcome and stimulating. He cannot subscribe to the frequency theory of probability, but adopts a new approach to the subjective theory by referring it back, like the economists, to a theory of utility. Even here the decision aspect of the subject affects his thinking, but the treatment is interesting and well written.

In founding statistical inference on minimax theory, Prof. Savage starts on the defensive; for the minimax principle, in its crude form, says that we must act as if the worst will happen, which is reasonable enough in games against a clever adversary but is not reasonable against Nature. Prof. Savage, however, extricates himself from this difficulty, not without a struggle, and proceeds to ground which is more familiar to the ordinary statistician by discussing estimation and tests of significance. He has some rather startling things to say about accepted theories on this subject, but what he says is well thought out and deserves attention. It is to be hoped that his criticisms are not lost to sight under the controversy which they may well raise.

M. G. KendaLl

\section{A HANDBOOK FOR MUSEUM CURATORS}

The Preservation of Natural History Specimens Edited and Compiled by Reginald Wagstaffe and Dr. J. Havelock Fidler. Vol. 1: Invertebrates. Pp. xiii +205 . (London: H. F. and G. Witherby, Ltd., 1955.) 42s, net.

$7 \mathrm{HE}$ intention of this book is to bring together in one place a collection of all those techniques concerned with the preservation of natural history specimens that have proved to be efficacious. In addition to the old and well-tried methods, a considerable amount of original work is included.

The first part of the book consists of a systematic survey of the chief invertebrate groups from the Protozoa to the Tunicata. In each group there is given the method of killing (preceded by narcotization where necessary), of fixing and of preserving. In cases where special manipulation is necessary, this is described and sometimes illustrated with a diagram. For the detailed formulæ in each case the reader is referred to the appropriate section of the appendix. This saves an intolerable amount of repetition.

Clearly, there are groups which lend themselves to fuller treatment than others, and the Insecta are dealt with in considerable detail, particularly as to setting and mounting. One suspects that the beauty of Miss Begg's drawings has beguiled the editors into extending this section a little beyond the limits of necessity. In the section on Fchinodermatia no mention is made of the corrosive sublimate method of preserving the test, particularly of Echinus, recommended by the late R. T. Jackson, of Harvard (see Mus. J., 29; May 1930). This method preserves both the spines and the lantern and is exceedingly simple to apply. It is also useful in the case of many Crustacea.

It may be noted that throughout the book the emphasis is upon the preservation of the external features. Anatomical dissection and display are not considered.

The second part of the book consists of five appendixes dealing with apparatus and instruments ; reagents; labels and labelling; storage; and microscopy. Here there is a large and useful collection of formulæ and recipes, and the description of many pieces of apparatus, both simple and ingenious. Many of them are well known; some of them are new. It is perhaps the most useful section of the book. Among the formulæ for cements for sealing museum jars, the asphalt-guttapercha mixture is recommended, particularly for jars containing formalin, but it is equally effective for 70 per cent alcohol. I have found it quite the best generalpurpose cement for museum work. Stitching on to cards is recommended in a number of cases. For wet mounts, stitching on to glass is perhaps the best. method, and a later edition might include a simple technique for drilling holes in glass (see Mus. .. , 35 ; Nov. 1935); or maybe it will appear in Vol. 2.

In a work of reference, the importance of the index is obvious. In this case it performs its job efficiently and with the minimum of irritation. The general format of the book is excellent. The binding is strong, the paper good, the type clear, and Miss Begg's illustrations a real embellishment. The editors are to be congratulated on a very sound job, and one looks forward to the appearance of the second volume.
W. Eric Stoneman 